\title{
Habilidades lingüísticas y comprensión lectora en la oquedad del siglo XXI: una mirada a la Institución Educativa Politécnica de Puno - Perú
}

\author{
Language skills and reading comprehension in the empty of the 21st century: a look at the \\ Polytechnic Educational Institution of Puno - Peru \\ Competências linguísticas e compreensão da leitura no vazio do século XXI: um olhar sobre a \\ Instituição Politécnica de Puno - Peru
}

\author{
Jesús Wiliam Huanca-Arohuanca ${ }^{1}$ \\ jhuancaar@unsa.edu.pe \\ https://orcid.org/0000-0002-7353-1166 \\ Dometila Mamani Jilaja² \\ domamani@unap.edu.pe \\ https://orcid.org/0000-0003-2357-8684
Pedro Carlos Huayanca Medina ${ }^{2}$
phuayanca@unap.edu.pe \\ https://orcid.org/0000-0002-7873-8510
}

\author{
Meredith Lency Asqui Manzano² \\ meredith.asqui@gmail.com \\ https://orcid.org/0000-0003-0467-6772
}

\author{
Humberto Mamani-Coaquira² \\ hmamanic@unap.edu.pe \\ https://orcid.org/0000-0002-0569-860X
}

\author{
Francisco Charaja Cutipa ${ }^{2}$ \\ franciscocharaja06@gmail.com \\ https://orcid.org/0000-0002-9511-9332
}

\section{${ }^{1}$ Universidad Nacional de San Agustín de Arequipa, Perú 2Universidad Nacional del Altiplano, Perú}

\section{RESUMEN}

El propósito de la investigación es analizar la relación entre habilidades lingüísticas y la comprensión de lectura en los estudiantes de la Institución Educativa Politécnica de Puno. Siendo el diseño de investigación el enfoque cuantitativo no experimental, con un universo poblacional de 240 estudiantes distribuidos en cinco grados y con una muestra de 148 estudiantes de tipo intencional probabilístico. Para la recolección de datos se utilizó técnicas de observación complementado con la evaluación de la comprensión de lectura procesado con SPSS. Por otro lado, los instrumentos utilizados fueron: la ficha de observación de las habilidades lingüísticas y la prueba de comprensión de textos, el cual consta de tres niveles: literal, inferencial y crítico. En conclusión, se indica que los niveles de desarrollo de las habilidades lingüísticas evidenciaron un grado de covariación positivo considerable (Rho $=0,616^{*}$ ) con relación a la comprensión de lectura representado en un $61.6 \%$, todo ello, sustentado con los estadígrafos obtenidos que permitieron afirmar la eficacia de la técnica aplicada.

Palabras clave: Educación; Enseñanza y Aprendizaje; Escuela; Conocimiento; Formación

\section{ABSTRACT}

The purpose of the research is to analyze the relationship between language skills and reading comprehension in students of the Puno Polytechnic Educational Institution. The research design being the non-experimental quantitative approach, with a population universe of 240 students distributed in five grades and with a sample of 148 students of intentional probabilistic type. For data collection, observation techniques were used complemented by the evaluation of reading comprehension processed with SPSS. On the other hand, the instruments used were: the observation sheet of linguistic skills and the proof of understanding of texts, which consists of three levels: literal, inferential and critical. In conclusion, it is indicated that the levels of development of linguistic skills showed a considerable degree of positive covariation (Rho s $0.616^{*}$ ) in relation to the reading comprehension represented by $61.6 \%$, all supported by the states that obtained that allowed to affirm the effectiveness of the applied technique.

Key words: Education; learning; school; knowledge; teaching and training

\section{RESUMO}

O objetivo da investigação é analisar a relação entre as competências linguísticas e a compreensão da leitura nos alunos da Instituição Politécnica de Puno. 0 desenho da investigação é a abordagem quantitativa não experimental, com um universo populacional de 240 alunos distribuídos em cinco graus e com uma amostra de 148 alunos de tipo probabilístico intencional. Foram utilizadas técnicas de observação complementadas pela avaliação da compreensão da leitura processada com SPSS para a recolha de dados. Por outro lado, os instrumentos utilizados foram: a folha de observação das competências linguísticas e a prova de compreensão dos textos, que consistem em três níveis: literal, inferencial e crítico. Em conclusão, é indicado que os níveis de desenvolvimento das competências linguísticas apresentaram um grau considerável de covariação positiva (Rho x 0,616*) em relação à compreensão da leitura representada em $61,6 \%$, todos suportados pelos parâmetros obtidos que permitiram afirmar a eficácia da técnica aplicada.

Palavras-chave: Educação; aprendizagem; escola; conhecimento; ensino e formação 


\section{INTRODUCCCIÓN}

Perú es un país que muestra un crecimiento macroeconómico significativo, pero eso no ha disminuido las brechas de desigualdad existentes en el país (Canaza-Choque y HuancaArohuanca, 2019; Huanca-Arohuanca, 2019; 2020c; Velázquez et al., 2017) ni mucho menos ha mejorado la calidad educacional en cuanto a la comprensión de lectora y otras variables igual de importantes. En esa medida, el ex ministro de educación, Jaime Saavedra, manifestaba que en la actualidad en los países de ingreso bajo y medio, el 53\% de los estudiantes a los 10 años no puede leer ni entender un texto simple, a pesar de que en su gran mayoría tienen acceso a la educación. A ese indicador lo considera como pobreza de aprendizajes. Así mismo, sustenta que en Perú, ese número es 56\% (Saavedra, 2020). Lo cual lleva a decir que, la realidad de la comprensión lectora en Perú es pobre y aún hay mucho camino por recorrer. También se señala que según la última Evaluación Censal de Estudiantes (ECE) llevada a cabo a fines de 2018, indica que en el caso de primaria el $34.8 \%$ de la población nacional que cursa el cuarto grado comprende lo que lee, mientras que en el caso de los alumnos de segundo grado de secundaria solo el $16.2 \%$ comprenden lo que leen (Economía y Negocio, 2019). De manera que, los indicadores muestran el problema real de la educación peruana impregnado en todas sus esferas, desde el nivel más alto hasta el nivel más bajo referido evidentemente a docentes $\mathrm{y}$ estudiantes.

Cabe señalar que, de los 511,000 alumnos del segundo grado de educación secundaria, la región Tacna lidera la lista junto a Arequipa y Moquegua en los resultados de la ECE 2019, en ese criterio, Tacna se reconfigura positivamente en las siguientes áreas; en ciencia y tecnología, obtuvo un $18.6 \%$ de nivel satisfactorio; en matemática lograron un $38.0 \%$ de nivel satisfactorio; y en el área de lectura, obtuvieron un $27.0 \%$ de nivel satisfactorio (Andina, 2020). Lo cual indica que, ni con las regiones en los que la educación aparentemente es de un nivel significativo, los resultados no pasan el 50\% de satisfacción. Ahora bien, si hablamos de la región altiplánica, está ni siquiera aparece en las noticias y las opiniones de los expertos dado a su pobre rendimiento.

Teniendo claro la gravedad del asunto, es que retomar la gramática generativa de Noam Chomsky conocida en la actualidad como biolingüística dedicado a postular la existencia de una estructura mental innata que permite la producción y comprensión de cualquier enunciado en cualquier idioma natural (Bustamante, 2010; Barón y Müller, 2014), resulta esencial para comprender bien las habilidades lingüísticas y la comprensión de lectura, ya que, una gran parte de la información que se transmite a los alumnos se presenta por escrito. Así, un alumno no entiende una lección ni puede realizar un trabajo si no es capaz de comprender lo que lee. De otra parte, comprender los argumentos de otros ayudaría al alumno a construir los suyos, con mayor lógica y eficacia. Ese dominio es lo que se valora por los profesores en la medición del rendimiento del alumno (Mata et al., 2007).

En la región Puno, los colegios en general no desarrollan el conjunto de conocimientos, habilidades y destrezas que requiere el uso adecuado, correcto, coherente y estético tanto del código oral como del escrito, centrándola en escuchar y hablar, leer y escribir de forma competente (Reyzábal, 2012). Ya que la mayoría de los docentes presenta precariedades en pedagogía, potencial cognitivo $\mathrm{y}$ otras habilidades relacionadas al aspecto psicológico. Los que enseñan aun no asimilan que los niños se inician en el aprendizaje de la 
Jesús Wiliam Huanca-Arohuanca; Meredith Lency Asqui Manzano; Dometila Mamani Jilaja;

Humberto Mamani-Coaquira; Pedro Carlos Huayanca Medina, y Francisco Charaja Cutipa

lectura en un sistema alfabético como el Aymara-Quechua (Huanca-Arohuanca, 2020a; 2020b: 2020c; 2021; Huanca-Arohuanca y Geldrech, 2020; Huanca-Arohuanca y Pilco, 2021) y el castellano, sabiendo que a partir de ahí se produce una relación directa entre el lenguaje oral y escrito, puesto que nuestro sistema de representación está centrado en la segmentación de la cadena hablada, y además han de ser conocedores de que las letras son signos gráficos que se corresponden con los elementos sonoros (Gutiérrez, 2018).

Tomando en cuenta la problemática en los acápites precedentes, es que se pone a flote el desarrollo de las cuatro habilidades lingüísticas (saber hablar, saber leer, saber escribir y saber escuchar), los cuales podrían contribuir en cierta medida a lograr una comprensión de lectura adecuada. Así como también, podrían favorecer al uso de un lenguaje apropiado en diversas situaciones sociales como; destrezas de reflexión, la toma de conciencia de las propias capacidades y recursos, la memorización, la argumentación y la síntesis de las ideas propias $\mathrm{y}$ ajenas que favorecen el aprendizaje de los estudiantes. Finalmente, un desarrollo adecuado de las habilidades lingüísticas podría contribuir decisivamente al desarrollo competente del estudiante; entendida como conjunto de destrezas para las relaciones sociales, la convivencia, formación integral, el aprendizaje significativo y el entendimiento entre una determinada comunidad educativa y la sociedad en general.

\section{MÉTODO}

El artículo se desdobló dentro del enfoque cuantitativo descriptivo-interpretativo (Huanca-Arohuanca, Escobar-Mamani, et al., 2020; Huanca-Arohuanca, Butrón, et al., 2020) de tipo no experimental. Puesto que, a partir de las aportaciones de la investigación sobre el tema, se planteó la siguiente interrogante: ¿cuál es el grado de correlación entre el nivel de desarrollo de las habilidades lingüísticas y la comprensión de lectura en los estudiantes de la Institución Educativa Secundaria (IES) "Politécnico Huáscar" de Puno en el año escolar 2018? Para dar respuesta a esta cuestión se evaluó, en grupos determinados de alumnos, tanto las habilidades lingüísticas como la comprensión lectora, para luego relacionar los datos obtenidos (Mata et al., 2007).

El universo poblacional estuvo conformado por 240 estudiantes del $1^{\circ}$ a $5^{\circ}$ grado de la IES "Politécnico Huáscar" de la región Puno. Además, la muestra situacional con el que se trabajó fue de 148 estudiantes entre varones (71) y mujeres (77), tal como se evidencia en la siguiente Tabla 1.

Tabla 1. Muestra de estudio.

\begin{tabular}{lcccc}
\hline \multicolumn{1}{c}{ Grados } & Varones & Mujeres & f & \% \\
\hline Primer grado & 12 & 16 & 28 & 19 \\
Segundo grado & 18 & 14 & 32 & 22 \\
Tercer Grado & 16 & 13 & 29 & 20 \\
Cuarto grado & 8 & 18 & 26 & 17 \\
Quinto grado & 17 & 16 & 33 & 22 \\
\hline Total & $\mathbf{7 1}$ & $\mathbf{7 7}$ & $\mathbf{1 4 8}$ & $\mathbf{1 0 0}$ \\
\hline
\end{tabular}

Fuente: Nómina de estudiantes del campo de estudio. 
Jesús Wiliam Huanca-Arohuanca; Meredith Lency Asqui Manzano; Dometila Mamani Jilaja;

Humberto Mamani-Coaquira; Pedro Carlos Huayanca Medina, y Francisco Charaja Cutipa

Las técnicas empleadas fueron la observación la cual se utilizó para recoger los datos correspondientes a la variable "habilidades lingüísticas". La observación según Hernández y otros (2014) consiste en el registro sistemático, válido y confiable del comportamiento o de la conducta manifiesta, la cual puede utilizarse en muy diversas circunstancias. Otra técnica fue la evaluación de la comprensión de lectura en donde Cárdenas-Espinoza y Guevara-Benítez (2013) manifiestan que este método consiste en una técnica de evaluación semi objetiva que requiere la elaboración de una respuesta breve. La construcción de la respuesta reflejó la mejor forma la comprensión que tienen los alumnos, ya que debieron activar los procesos mentales de tipo literal, inferencial y crítico para relacionar la información explícita del texto con su conocimiento previo.

En cuento a los instrumentos que fueron usados se encuentran la Ficha de observación: El instrumento consiste en registrar la descripción detallada de lugares, personas, hechos, etc., que forman parte de la investigación. Dicho de otra manera, la ficha de observación es un instrumento de investigación, evaluación y recolección de datos; referido a un objetivo específico, en el que se determinan variables explícitas (Gutierrez y Sanz, 2018). El instrumento se utilizó para registrar datos correspondientes a las cuatro habilidades lingüísticas (saber hablar, saber leer, saber escribir y saber escuchar), mientras se desarrollaban las distintas sesiones de aprendizaje, en actividades como:

- Saber hablar: exposiciones, debates, participaciones orales y opiniones.

- Saber leer: lectura en voz alta, lectura silenciosa, lectura literal, lectura mecánica, lectura comprensiva y lectura crítica.

- Saber escribir: redacción de textos narrativos, textos poéticos, textos informativos, textos argumentativos $\mathrm{y}$ elaboración de resúmenes.

- Saber escuchar: comprensión del mensaje cuando el docente expone el tema, opiniones en base a las exposiciones de sus compañeros, comprensión de los audios y videos, escucha activa cuando los alumnos leen en voz alta, etc.

Es importante mencionar que dicho el instrumento de registro fue adaptado de otro instrumento denominado "Ficha de Observación sobre el performance de las habilidades sociales", cuya fuente es la Oficina General del Sistema de Bibliotecas y Biblioteca Central UNMSM.

También se usó la Prueba de comprensión de lectura: El instrumento consta de dos subtest: Subtest Texto Base y Subtest Modelo de Situación. El Subtest Texto Base, evalúa la habilidad de los alumnos para comprender las ideas expresamente señaladas en el texto, es decir, contiene preguntas de nivel literal (4 preguntas). El Subtest Modelo de Situación, evalúa la habilidad de los alumnos para relacionar las ideas del texto con su conocimiento previo o elaborar nuevas ideas a partir del texto leído, así como también, dar a conocer el punto de vista que posee en base a un texto determinado. Es más, contiene preguntas inferenciales (4 preguntas) y preguntas de nivel crítico (2 preguntas) (Ramos, 2006). 


\section{Procedimiento de recolección de datos}

- Coordinación con las autoridades correspondientes para la autorización respectiva.

- Elaboración de los instrumentos de investigación.

- Aplicación de los instrumentos de investigación.

- Organización de los datos recogidos.

\section{Plan de análisis de interpretación de datos}

Para realizar el análisis e interpretación de los resultados se empleó las siguientes herramientas estadísticas:

- Tablas de distribución de frecuencias.

- Gráficos estadísticos.

- Hipótesis estadístico: hipótesis alterna e hipótesis nula.

- Contrastación de las hipótesis.

\section{Diseño estadístico para la prueba de hipótesis}

Para conocer la correlación entre las variables de investigación, se utilizó la fórmula de coeficiente de correlación de Spearman y esta a su vez, tuvo como requisito fundamental comprobar el Supuesto de Normalidad para datos de la variable cuantitativa; es por esa razón que se optó por realizar la prueba de KolmogorovSmirnov de contraste para la normalidad, más conocida como una prueba de bondad de ajuste, es decir, todo ello, sirvió para verificar si las puntuaciones que se ha obtenido de la muestra siguen o no una distribución normal (García Bellido et al., 2010; Hernández et al., 2014). Puesto que, su objetivo es señalar si los datos provienen de una población que tiene la distribución teórica especificada, es decir, lo que hace es contrastar si las observaciones podrían razonablemente proceder de la distribución especificada. Para ello, se tiene en cuenta lo siguiente:

a) Hipótesis de la normalidad:

$\mathbf{H}_{\mathbf{0}}$ : Los datos siguen la distribución normal

$\mathbf{H}_{\mathbf{a}}$ : Los datos no siguen la distribución normal

b) El nivel de significancia de probabilidad es: 0,01 .

El coeficiente de correlación de rangos de Spearman es de gran utilidad en aquellos análisis de datos en donde se desea conocer la relación lineal entre variables, cuyas escalas de medidas sean al menos ordinales, o que exista suficientes evidencias de que las variables en estudio a pesar de ser cuantitativas no siguen un comportamiento normal (Hernández et al., 2014). Puesto que, el coeficiente de correlación rho de Spearman es el mismo coeficiente de correlación de Pearson, pero aplicado luego de efectuar una transformación de los valores originales de las variables en rangos, tomando valores entre $-1 \mathrm{y}+1 \mathrm{y}$ se interpreta de la misma manera que el de Pearson. Este coeficiente se denota comúnmente como $\mathrm{r}_{\mathrm{S}}$ (Martínez et al., 2009).

Por su parte, Pérez (2005) señala que cuando se determina a partir de las variables cuantitativas, el grado de asociación lineal obtenido no es el de los valores de las variables, sino el de las clasificaciones por rangos de dichos valores. Ese indicador también es llamado coeficiente de correlación por rangos, entendiéndose por rango de un 
Jesús Wiliam Huanca-Arohuanca; Meredith Lency Asqui Manzano; Dometila Mamani Jilaja;

Humberto Mamani-Coaquira; Pedro Carlos Huayanca Medina, y Francisco Charaja Cutipa

valor de una variable, el lugar que ocupa dicho valor en el conjunto total de valores de la variable, suponiendo de antemano que los valores se tienen ordenados de menor a mayor o viceversa. Ese coeficiente se basa en la concordancia o discordancia de las clasificaciones por rangos de las modalidades de las variables cualitativas estudiadas.

Algebraicamente, el coeficiente de correlación de Spearman se obtiene como: Sean $X$ e $Y$ dos variables aleatorias cualitativas ordinales tomadas de una muestra de tamaño $n$, con categorías $\mathrm{A}_{\mathrm{i}}$ y $\mathrm{B}_{\mathrm{i}}$, $\mathrm{y}$ sean $\mathrm{X}_{\mathrm{i}}$ e $\mathrm{Y}_{\mathrm{i}}$ los rangos que les corresponden a $\mathrm{A}_{\mathrm{i}}$ y $\mathrm{B}_{\mathrm{i}}$, entonces, para Escurra (1998) es:

$$
r_{S}=1-\frac{6 \cdot \sum_{i=1}^{n} d_{i}^{2}}{n^{3}-n}
$$

Donde: $\mathbf{d}_{\mathbf{i}}=\mathrm{x}_{\mathrm{i}}-\mathrm{y}_{\mathrm{i}}(\mathrm{i}=1,2, \mathrm{n} ; \mathrm{n}=$ Nro. de parejas de rangos). Será $\mathbf{r}_{\mathbf{s}}=+1$ cuando la concordancia entre los rangos es perfecta por lo tanto $\mathbf{d i}=\mathrm{x}_{\mathrm{i}}-\mathrm{y}_{\mathrm{i}}=0$, mientras que será $\mathbf{r}_{\mathrm{s}}=$ 1 cuando la discordancia es perfecta y cuando no existe concordancia y discordancia entonces $\mathbf{r}_{\mathrm{s}}=0$.

Tabla 2. Grado de relación según coeficiente de correlación.

\begin{tabular}{ll}
\hline \multicolumn{1}{c}{ Rango } & \multicolumn{1}{c}{ Relación } \\
\hline$-0.91 \mathrm{a}-1.00$ & Correlación negativa perfecta \\
$-0.76 \mathrm{a}-0.90$ & Correlación negativa muy fuerte \\
$-0.51 \mathrm{a}-0.75$ & Correlación negativa considerable \\
$-0.11 \mathrm{a}-0.50$ & Correlación negativa media \\
$-0.01 \mathrm{a}-0.10$ & Correlación negativa débil \\
0.00 No existe & Correlación \\
$+0.01 \mathrm{a}+0.10$ & Correlación positiva débil \\
$+0.11 \mathrm{a}+0.50$ & Correlación positiva media \\
$+0.51 \mathrm{a}+0.75$ & Correlación positiva considerable \\
$+0.76 \mathrm{a}+0.90$ & Correlación positiva muy fuerte \\
$+0.91 \mathrm{a}+1.00$ & Correlación positiva perfecta \\
\hline
\end{tabular}

Fuente: (Hernández et al., 1991).

\section{RESULTADOS}

En la sección se presentan los resultados obtenidos de la investigación que corresponde a la determinación de la relación o asociación existente entre el nivel de desarrollo de las habilidades lingüísticas y la comprensión de lectura de la siguiente manera; en primer lugar, se empieza planteando los estadísticos descriptivos correspondientes a cada variable, por consiguiente, se presentan los resultados de la variable "El nivel de desarrollo de las habilidades lingüísticas"; luego, los resultados de la variable "Comprensión de lectura"; y, posteriormente el análisis de la correlación de las variables, según el coeficiente de correlación de Spearman. 
Jesús Wiliam Huanca-Arohuanca; Meredith Lency Asqui Manzano; Dometila Mamani Jilaja;

Humberto Mamani-Coaquira; Pedro Carlos Huayanca Medina, y Francisco Charaja Cutipa

Tabla 3. Estadísticos descriptivos de las habilidades lingüísticas y la comprensión de lectura.

\begin{tabular}{|c|c|c|c|c|}
\hline \multicolumn{5}{|c|}{ DATOS DESCRIPTIVOS } \\
\hline & & & Estadístico & Desv. Error \\
\hline \multirow{13}{*}{$\begin{array}{l}\text { Habilidades } \\
\text { lingüísticas }\end{array}$} & \multicolumn{2}{|l|}{ Media } & 14,74 & \multirow{13}{*}{$\begin{array}{l}0,199 \\
0,396\end{array}$} \\
\hline & $95 \%$ de intervalo & Límite inferior & 14,45 & \\
\hline & \multicolumn{2}{|c|}{ la media } & 15,03 & \\
\hline & \multicolumn{2}{|c|}{ Media recortada al 5\% } & 14,87 & \\
\hline & \multicolumn{2}{|c|}{ Mediana } & 15,00 & \\
\hline & \multicolumn{2}{|l|}{ Varianza } & 3,199 & \\
\hline & \multicolumn{2}{|l|}{ Desviación } & 1,789 & \\
\hline & \multicolumn{2}{|l|}{ Mínimo } & 8 & \\
\hline & \multicolumn{2}{|l|}{ Máximo } & 18 & \\
\hline & \multicolumn{2}{|l|}{ Rango } & 10 & \\
\hline & \multicolumn{2}{|l|}{ Rango intercuartil } & 2 & \\
\hline & \multicolumn{2}{|l|}{ Asimetría } & $-1,060$ & \\
\hline & \multicolumn{2}{|l|}{ Curtosis } & 2,507 & \\
\hline \multirow{13}{*}{$\begin{array}{l}\text { Comprensión } \\
\text { de lectura }\end{array}$} & \multicolumn{2}{|l|}{ Media } & 12,93 & \multirow[t]{11}{*}{0,223} \\
\hline & 95\% de intérvalo & Límite inferior & 12,49 & \\
\hline & $\begin{array}{l}\text { de confianza para } \\
\text { la media }\end{array}$ & Límite superior & 13,37 & \\
\hline & \multicolumn{2}{|c|}{ Media recortada al 5\% } & 13,02 & \\
\hline & \multicolumn{2}{|c|}{ Mediana } & 14,00 & \\
\hline & \multicolumn{2}{|l|}{ Varianza } & 7,370 & \\
\hline & \multicolumn{2}{|l|}{ Desviación } & 2,715 & \\
\hline & \multicolumn{2}{|l|}{ Mínimo } & 4 & \\
\hline & \multicolumn{2}{|l|}{ Máximo } & 18 & \\
\hline & \multicolumn{2}{|l|}{ Rango } & 14 & \\
\hline & \multicolumn{2}{|l|}{ Rango intercuartil } & 2 & \\
\hline & \multicolumn{2}{|l|}{ Asimetría } &,- 432 & 0,199 \\
\hline & \multicolumn{2}{|l|}{ Curtosis } & 0,435 & 0,396 \\
\hline
\end{tabular}

Fuente: Paquete estadístico para las Ciencias Sociales (SSPS).

Nivel de desarrollo de las habilidades lingüísticas

Para determinar el desarrollo o no de las habilidades lingüísticas (saber hablar, saber escribir, saber leer y saber escuchar) en los estudiantes, se aplicó una ficha de observación durante varias sesiones de aprendizaje. La ficha de observación fue calificada de acuerdo a la matriz de ponderación prevista. Los datos fueron organizados en una Tabla de distribución porcentual, lo que se muestra a continuación: 


\section{Habilidad lingüística al momento de hablar}

Tabla 4. Habilidad lingüística al momento de hablar.

\begin{tabular}{|c|c|c|c|c|c|c|c|c|c|c|c|}
\hline \multicolumn{12}{|c|}{ ESCALA DE MEDICIÓN } \\
\hline \multicolumn{2}{|c|}{$\begin{array}{l}\text { Pésimo } \\
(01-05)\end{array}$} & \multicolumn{2}{|c|}{$\begin{array}{c}\text { Deficiente } \\
(06-10)\end{array}$} & \multicolumn{2}{|c|}{$\begin{array}{c}\text { Regular } \\
(11-13)\end{array}$} & \multicolumn{2}{|c|}{$\begin{array}{c}\text { Bueno } \\
(14-17)\end{array}$} & \multicolumn{2}{|c|}{$\begin{array}{c}\text { Excelente } \\
(18-20)\end{array}$} & \multicolumn{2}{|c|}{ TOTAL } \\
\hline $\mathrm{f}$ & $\%$ & $\mathrm{f}$ & $\%$ & $\mathrm{f}$ & $\%$ & $\mathrm{f}$ & $\%$ & $\mathrm{f}$ & $\%$ & $\mathrm{f}$ & $\%$ \\
\hline 0 & 0.0 & 9 & 6.1 & 46 & 31.1 & 85 & 57.4 & 8 & 5.4 & 148 & 100 \\
\hline
\end{tabular}

Fuente: Ficha de observación.

En la Tabla 4, se presentan los resultados sobre el nivel de desarrollo de la habilidad lingüística al momento de hablar de los estudiantes de la IES "Politécnico Huáscar" de Puno, de un total de 148 estudiantes investigados, mientras desarrollaban distintas actividades como exposiciones, debates, participaciones orales y opiniones. Se puede observar que, 85 estudiantes, los cuales representan $57.4 \%$ evidenciaron que desarrollan su habilidad lingüística denominada saber hablar de manera buena; es decir, en base a la ficha de observación que se les aplicó los estudiantes obtuvieron puntajes comprendidos entre 14 a 17 puntos. A esta mayoría inclusive se suman aquellos estudiantes que desarrollan sus habilidades lingüísticas en el nivel regular, los cuales representan un $31.1 \%$ del total de alumnos. Por otro lado, solo 9 estudiantes (6.1\%) logran resultados deficientes según el desarrollo de su habilidad lingüística al momento de hablar. Además, se evidencia que tan solo 8 estudiantes (5.4\%) obtuvieron un puntaje que según la escala de medición se considera excelente.

De tal manera, se infiere que la mayoría de los estudiantes desarrollan su habilidad lingüística (saber hablar) de manera buena en las respectivas sesiones de aprendizaje, es decir, los alumnos al momento de la expresión oral demuestran fluidez, un vocabulario rico y abundante $\mathrm{y}$ sobre todo credibilidad $\mathrm{y}$ confianza.

\section{Habilidad lingüística al momento de escribir}

Tabla 5. Habilidad lingüística al momento de escribir.

\begin{tabular}{|c|c|c|c|c|c|c|c|c|c|c|c|}
\hline \multicolumn{12}{|c|}{ ESCALA DE MEDICIÓN } \\
\hline \multicolumn{2}{|c|}{$\begin{array}{l}\text { Pésimo } \\
\text { (01-05) }\end{array}$} & \multicolumn{2}{|c|}{$\begin{array}{c}\text { Deficiente } \\
(06-10)\end{array}$} & \multicolumn{2}{|c|}{$\begin{array}{l}\text { Regular } \\
(11-13)\end{array}$} & \multicolumn{2}{|c|}{$\begin{array}{c}\text { Bueno } \\
(14-17)\end{array}$} & \multicolumn{2}{|c|}{$\begin{array}{l}\text { Excelente } \\
(18-20)\end{array}$} & \multicolumn{2}{|c|}{ TOTAL } \\
\hline $\mathrm{f}$ & $\%$ & $\mathrm{f}$ & $\%$ & $\mathrm{f}$ & $\%$ & $\mathrm{f}$ & $\%$ & $\mathrm{f}$ & $\%$ & $\mathrm{f}$ & $\%$ \\
\hline 0 & 0.0 & 8 & 5.4 & 41 & 27.7 & 89 & 60.1 & 10 & 6.8 & 148 & 100 \\
\hline
\end{tabular}

Fuente: Ficha de observación. 
Jesús Wiliam Huanca-Arohuanca; Meredith Lency Asqui Manzano; Dometila Mamani Jilaja;

Humberto Mamani-Coaquira; Pedro Carlos Huayanca Medina, y Francisco Charaja Cutipa

En la Tabla 5 se presentan los resultados sobre el nivel de desarrollo de la habilidad lingüística al momento de escribir. Pues de un total de 148 estudiantes (100\%) investigados en distintas sesiones de aprendizaje, se observó la redacción de textos narrativos, textos poéticos, textos informativos, textos argumentativos y elaboración de resúmenes.

De esa forma, se puede observar que 8 alumnos, los cuales representan el 5.4\% evidencian una escala deficiente; 41 alumnos (20.6\%), una escala regular; 89 alumnos que representa el $60.1 \%$, una escala buena; frente a 10 alumnos (12.7\%) que evidencian una escala excelente. Esos resultados indican que la mayoría de los estudiantes (60.1\%) se caracterizan por poseer capacidades para planificar las ideas, organizar las ideas y redactarlas en el proceso que escriben sus textos.

En su actividad de aprendizaje, resaltan por su cualidad de generar ideas que conformarán el texto que desea escribir y, luego, lo representa en su mente. Una vez determinado ese contenido, el estudiante lo textualiza; para ello utiliza sus competencias comunicacionales y redaccionales con las cuales elabora en su mente las estructuras lingüísticas necesarias para transmitir las ideas e intenciones que desea comunicar. Finalmente, se aboca a leer repetidamente lo escrito, manteniendo una actitud crítica, para luego ajustar las partes que le parecen insatisfactorias.

\section{Habilidad lingüística al momento de leer}

Tabla 6. Habilidad lingüística al momento de leer.

\begin{tabular}{|c|c|c|c|c|c|c|c|c|c|c|c|}
\hline \multicolumn{12}{|c|}{ ESCALA DE MEDICIÓN } \\
\hline \multicolumn{2}{|c|}{$\begin{array}{l}\text { Pésimo } \\
(01-05)\end{array}$} & \multicolumn{2}{|c|}{$\begin{array}{c}\text { Deficiente } \\
(06-10)\end{array}$} & \multicolumn{2}{|c|}{$\begin{array}{c}\text { Regular } \\
(11-13) \\
\end{array}$} & \multicolumn{2}{|c|}{$\begin{array}{c}\text { Bueno } \\
(14-17) \\
\end{array}$} & \multicolumn{2}{|c|}{$\begin{array}{c}\text { Excelente } \\
(18-20) \\
\end{array}$} & \multicolumn{2}{|c|}{ TOTAL } \\
\hline $\mathrm{f}$ & $\%$ & $\mathrm{f}$ & $\%$ & $\mathrm{f}$ & $\%$ & $\mathrm{f}$ & $\%$ & $\mathrm{f}$ & $\%$ & $\mathrm{f}$ & $\%$ \\
\hline 0 & 0 & 18 & 12.2 & 49 & 33.1 & 61 & 41.2 & 20 & 13.5 & 148 & 100 \\
\hline
\end{tabular}

Fuente: Ficha de observación.

En la Tabla 6, se presentan los resultados sobre el nivel de desarrollo de la habilidad lingüística al momento de leer en los estudiantes de Puno, de un total de 148 estudiantes $(100 \%)$ investigados, se realizaron actividades como la lectura en voz alta, lectura silenciosa, lectura literal, lectura mecánica, lectura comprensiva y lectura crítica. Se puede observar que, 61 estudiantes los cuales representan $41.2 \%$ evidenciaron que desarrollan su habilidad lingüística denominada saber leer de manera buena; es decir, en base a la ficha de observación que se les aplicó, los estudiantes obtuvieron puntajes comprendidos entre 14 a 17 puntos. A esta mayoría inclusive se suman 49 estudiantes que desarrollan su habilidad lingüística (saber leer) en el nivel regular, los cuales representan un $33.1 \%$ del total de alumnos. Por otro lado, 18 estudiantes $(12.2 \%)$ lograron resultados deficientes según el desarrollo de su habilidad lingüística al momento de leer. Además, se evidencia que 20 estudiantes (13.5\%) obtuvieron un puntaje que según la escala de medición se considera excelente. 
Jesús Wiliam Huanca-Arohuanca; Meredith Lency Asqui Manzano; Dometila Mamani Jilaja;

Humberto Mamani-Coaquira; Pedro Carlos Huayanca Medina, y Francisco Charaja Cutipa

De tal manera, se infiere que la mayoría de los estudiantes desarrollan su habilidad lingüística (saber leer) de manera buena en las respectivas sesiones de aprendizaje, en otras palabras, los alumnos al momento de la lectura poseen la capacidad para comprender e interpretar los diferentes tipos de textos que se utilizan en las distintas sesiones de aprendizaje.

\section{Habilidad lingüística al momento de escuchar}

Tabla 7. Habilidad lingüística al momento de escuchar.

\begin{tabular}{|c|c|c|c|c|c|c|c|c|c|c|c|}
\hline \multicolumn{12}{|c|}{ ESCALA DE MEDICIÓN } \\
\hline \multicolumn{2}{|c|}{$\begin{array}{l}\text { Pésimo } \\
(01-05)\end{array}$} & \multicolumn{2}{|c|}{$\begin{array}{c}\text { Deficiente } \\
(06-10)\end{array}$} & \multicolumn{2}{|c|}{$\begin{array}{l}\text { Regular } \\
(11-13)\end{array}$} & \multicolumn{2}{|c|}{$\begin{array}{c}\text { Bueno } \\
(14-17)\end{array}$} & \multicolumn{2}{|c|}{$\begin{array}{l}\text { Excelente } \\
(18-20) \\
\end{array}$} & \multicolumn{2}{|c|}{ TOTAL } \\
\hline $\mathrm{f}$ & $\%$ & $\mathrm{f}$ & $\%$ & $\mathrm{f}$ & $\%$ & $\mathrm{f}$ & $\%$ & $\mathrm{f}$ & $\%$ & $\mathrm{f}$ & $\%$ \\
\hline 0 & 0.0 & 10 & 6.8 & 29 & 19.6 & 100 & 67.6 & 9 & 6.1 & 148 & 100 \\
\hline
\end{tabular}

Fuente: Ficha de observación.

En la Tabla 7, se presentan los resultados sobre el nivel de desarrollo de la habilidad lingüística al momento de escuchar. Entonces, de un total de 148 estudiantes (100\%) investigados en distintas sesiones de aprendizaje se observó variadas actividades como la comprensión del mensaje cuando el docente exponía un determinado tema, opiniones en base a las exposiciones de sus compañeros, comprensión de los audios y videos, escucha activa cuando los alumnos leen en voz alta, etc.

De esa forma, se puede observar que 10 alumnos que representan el $6.8 \%$ evidencian una escala deficiente; 29 alumnos (19.6\%), una escala regular; 100 alumnos que representan el 67.6\%, una escala buena; frente a 9 alumnos (6.1\%) que evidencian una escala excelente. Aquellos resultados indican que la mayoría de los estudiantes (67.6\%), los cuales obtuvieron un puntaje comprendido entre 14 a 17 puntos, se caracterizan por poseer capacidades para la comprensión e identificación del tema; así como también, el análisis y la reflexión de dicho tema oral.
En su actividad de aprendizaje, resaltan por su cualidad de demostrar una forma de comunicación donde el oyente entiende al hablante. En otras palabras, la habilidad de escuchar no sólo significa que los estudiantes comprendan e interpreten cuando se están expresando oralmente de un determinado tema, sino también cuando transmiten sus sentimientos, ideas o pensamientos. Por otro lado, si esta estrategia no se emplea (por parte del receptor) en un proceso de enseñanza aprendizaje, es imposible que éste se dé de forma favorable.

Con referente a las habilidades desarrolladas, la investigación realizada por Prieto (2012), relacionado con las habilidades o competencias lingüísticas en los estudiantes, también coinciden en que existe un buen desarrollo de las habilidades lingüísticas. Concretamente, ese investigador señala que en dicha investigación: se observa que existe un buen desarrollo en Comprensión Oral y Escrita (saber escuchar, saber hablar, saber escribir y saber leer). Esa cualidad es algo que debería ser utilizado por los docentes, por ser una de 
Jesús Wiliam Huanca-Arohuanca; Meredith Lency Asqui Manzano; Dometila Mamani Jilaja;

Humberto Mamani-Coaquira; Pedro Carlos Huayanca Medina, y Francisco Charaja Cutipa

las capacidades más estrechamente relacionadas con la lectoescritura.

Los resultados que aquí se muestran de alguna manera valida la concepción teórica que sostiene Madero y Gómez (2013), donde describe las relaciones existentes entre lenguaje oral y escrito en los años preescolares e identificar las habilidades concretas del lenguaje del niño preescolar que posibilitan la adquisición de la lectura y escritura convencionales.

En términos concluyentes, el objetivo de la investigación propuesto, se logra adecuadamente en el sentido como se ha planteado. Ya que, como objetivo se había propuesto identificar la escala de valoración en las que se ubican los estudiantes respecto al desarrollo de las habilidades lingüísticas. Ahora, los resultados que se han alcanzado permiten sostener que la escala en la que se ubican los estudiantes es buena. En cuanto se refiere a la hipótesis planteada, los resultados alcanzados permiten ratificar como cierta.

\section{Nivel de comprensión de lectura}

Para determinar el desarrollo de la comprensión de lectura en los estudiantes, se aplicó una prueba de comprensión lectora, luego, la prueba fue calificada de acuerdo a la ponderación prevista. De tal manera que, los datos se organizaron en una tabla de distribución porcentual, lo que se muestra seguidamente:

\section{Nivel literal}

Tabla 8. Comprensión de lectura en el nivel literal en los estudiantes de la IES "Politécnico Huáscar" de Puno.

\begin{tabular}{|c|c|c|c|c|c|c|c|c|c|c|c|}
\hline \multicolumn{12}{|c|}{ ESCALA DE MEDICIÓN } \\
\hline \multicolumn{2}{|c|}{$\begin{array}{l}\text { Pésimo } \\
\text { (01-05) }\end{array}$} & \multicolumn{2}{|c|}{$\begin{array}{l}\text { Deficiente } \\
(06-10)\end{array}$} & \multicolumn{2}{|c|}{$\begin{array}{l}\text { Regular } \\
(11-13)\end{array}$} & \multicolumn{2}{|c|}{$\begin{array}{c}\text { Bueno } \\
(14-17)\end{array}$} & \multicolumn{2}{|c|}{$\begin{array}{c}\text { Excelente } \\
(18-20)\end{array}$} & \multicolumn{2}{|c|}{ TOTAL } \\
\hline$f$ & $\%$ & $\mathrm{f}$ & $\%$ & $\mathrm{f}$ & $\%$ & $\mathrm{f}$ & $\%$ & $f$ & $\%$ & $\mathrm{f}$ & $\%$ \\
\hline 0 & 0.0 & 2 & 1.4 & 43 & 29.1 & 89 & 60.1 & 15 & 10.1 & 148 & 100 \\
\hline
\end{tabular}

Fuente: Ficha de observación.

En la Tabla 8, se presentan los resultados sobre el logro de la comprensión de lectura en el nivel literal de los estudiantes Puneños, donde se observa que, de los 148 alumnos investigados, 2 alumnos representan el 1.4\%, lo que evidencia una escala deficiente; 43 alumnos (29.1\%), una escala regular; 89 alumnos que representan el $60.1 \%$, que equivale a una escala buena; frente a 15 alumnos (10.1\%), que evidencian una escala excelente.
Dichos resultados indican que la mayoría de los estudiantes (60.1\%), obtuvieron un puntaje comprendido entre 14 a 17 puntos, así mismo, se identifican por poseer capacidades para extraer la información dada en el texto sin agregarle ningún valor interpretativo. Teniendo en cuenta que, los procesos fundamentales que conducen a este nivel de lectura son: observación, comparación, relación, clasificación, cambio, orden y las transformaciones. 
Jesús Wiliam Huanca-Arohuanca; Meredith Lency Asqui Manzano; Dometila Mamani Jilaja;

Humberto Mamani-Coaquira; Pedro Carlos Huayanca Medina, y Francisco Charaja Cutipa

\section{Nivel inferencial}

Tabla 9. Comprensión de lectura en el nivel inferencial en los estudiantes de la IES "Politécnico Huáscar" de Puno.

\begin{tabular}{|c|c|c|c|c|c|c|c|c|c|c|c|}
\hline \multicolumn{12}{|c|}{ ESCALA DE MEDICIÓN } \\
\hline \multicolumn{2}{|c|}{$\begin{array}{l}\text { Pésimo } \\
\text { (01-05) }\end{array}$} & \multicolumn{2}{|c|}{$\begin{array}{c}\text { Deficiente } \\
(06-10)\end{array}$} & \multicolumn{2}{|c|}{$\begin{array}{c}\text { Regular } \\
(11-13)\end{array}$} & \multicolumn{2}{|c|}{$\begin{array}{c}\text { Bueno } \\
(14-17)\end{array}$} & \multicolumn{2}{|c|}{$\begin{array}{c}\text { Excelente } \\
(18-20)\end{array}$} & \multicolumn{2}{|c|}{ TOTAL } \\
\hline $\mathrm{f}$ & $\%$ & $\mathrm{f}$ & $\%$ & $\mathrm{f}$ & $\%$ & $\mathrm{f}$ & $\%$ & $\mathrm{f}$ & $\%$ & $\mathrm{f}$ & $\%$ \\
\hline 5 & 3.4 & 8 & 5.4 & 55 & 37.2 & 76 & 51.4 & 4 & 2.7 & 148 & 100 \\
\hline
\end{tabular}

Fuente: Ficha de observación.

En la Tabla 9, se exhiben los resultados sobre el logro de la comprensión de lectura en el nivel inferencial en los estudiantes de la IES. "Politécnico Huáscar" de Puno, donde se observa que, de los 148 alumnos investigados, 5 alumnos (3.4\%) se encuentran en una escala pésima, 8 alumnos representan el 5.4\% evidenciando una escala deficiente; 55 alumnos (37.2\%) presentan una escala regular; 76 alumnos representan el 51.4\%, lo que indicaría una escala buena; frente a 4 alumnos $(2.7 \%)$ que se encuentran en una escala excelente.
Tales resultados indican que la mayoría de los estudiantes (51.4\%), obtuvieron un puntaje comprendido entre 14 a 17 puntos, es decir, se identifican por poseer capacidades o destrezas para deducir algo que no sea explícito en el texto que leen. Además, en este nivel de lectura amplían el nivel de análisis de un texto mediante la comprensión de la información que se les presenta. De tal modo, plantean inferencias o deducciones acerca de las ideas representativas localizadas en un determinado texto.

\section{Nivel crítico}

Tabla 10. Comprensión de lectura en el nivel crítico en los estudiantes de la IES "Politécnico Huáscar" de Puno.

\begin{tabular}{|c|c|c|c|c|c|c|c|c|c|c|c|}
\hline \multicolumn{12}{|c|}{ ESCALA DE MEDICIÓN } \\
\hline \multicolumn{2}{|c|}{$\begin{array}{l}\text { Pésimo } \\
(01-05)\end{array}$} & \multicolumn{2}{|c|}{$\begin{array}{c}\text { Deficiente } \\
(06-10) \\
\end{array}$} & \multicolumn{2}{|c|}{$\begin{array}{c}\text { Regular } \\
(11-13)\end{array}$} & \multicolumn{2}{|c|}{$\begin{array}{c}\text { Bueno } \\
(14-17) \\
\end{array}$} & \multicolumn{2}{|c|}{$\begin{array}{c}\text { Excelente } \\
(18-20) \\
\end{array}$} & \multicolumn{2}{|c|}{ TOTAL } \\
\hline f & $\%$ & $\mathrm{f}$ & $\%$ & $\mathrm{f}$ & $\%$ & $\mathrm{f}$ & $\%$ & $\mathrm{f}$ & $\%$ & $\mathrm{f}$ & $\%$ \\
\hline 3 & 2.0 & 12 & 8.1 & 23 & 15.5 & 100 & 67.6 & 10 & 6.8 & 148 & 100 \\
\hline
\end{tabular}

Fuente: Ficha de observación. 
Jesús Wiliam Huanca-Arohuanca; Meredith Lency Asqui Manzano; Dometila Mamani Jilaja;

Humberto Mamani-Coaquira; Pedro Carlos Huayanca Medina, y Francisco Charaja Cutipa

En la Tabla 10, se exhiben los resultados sobre el logro de la comprensión de lectura en el nivel crítico. Donde se observa que, de los 148 alumnos investigados, 3 alumnos (2.0\%) están en una escala pésima, 12 alumnos que representan el $8.1 \%$ evidencian una escala deficiente; 23 alumnos (15.5\%) se encuentran en una escala regular; 100 alumnos que representa el $67.6 \%$ están en una escala buena; frente a 10 alumnos (6.8\%) que evidencian una escala excelente.

Dichos resultados indican que la mayoría de los estudiantes (67.6\%) obtuvieron un puntaje comprendido entre 14 a 17 puntos, puesto que, se identifican por poseer capacidades o destrezas para la valoración y formación de juicios propios a partir de un determinado texto. Vale decir, elaboran argumentos para sustentar sus opiniones. Por lo que, en ese nivel de comprensión los estudiantes después de la lectura confrontarán el significado del texto con sus saberes y experiencias, luego, emitirán, un juicio crítico valorativo y expresivo (HuancaArohuanca y Canaza-Choque, 2019; HuancaArohuanca, Canaza-Choque, et al., 2020) de sus opiniones personales acerca de lo que leen.

Algo semejante sucede en la investigación realizada por Aliaga (2012), relacionado a la comprensión de lectura los estudiantes, que en la cual coincide en que existe un buen nivel de comprensión de lectura. Concretamente, este investigador señala que en dicha investigación: los resultados de la investigación demuestran la existencia de una relación positiva significativa $\left(\mathrm{r}=0,662^{* *}\right)$ entre ambas variables, destacando que los alumnos tienen buen nivel en la comprensión literal y un deficiente desempeño en la comprensión inferencial y crítico. Una tendencia similar es la que se aprecia en la presente investigación, dado que el $45 \%$ de los estudiantes logra un buen nivel de comprensión de lectura.

\section{La prueba de hipótesis}

La prueba de correlación de Spearman tiene como requisito fundamental realizar el Supuesto de Normalidad para datos de la variable cuantitativa. Es por esa razón que es necesario realizar la prueba de KolmogorovSmirnov de contraste para la normalidad:

Hipótesis de la normalidad:

$\mathbf{H}_{\mathbf{0}}$ : Los datos siguen la distribución normal

$\mathbf{H}_{\mathbf{a}}$ : Los datos no siguen la distribución normal

El nivel de significancia de probabilidad es: 0,01 .

La prueba de Kolmogorov-Smirnov:

Tabla 11. Prueba de normalidad con la prueba de Kolmogorov Smirnov.

\begin{tabular}{lrll}
\hline & \multicolumn{2}{c}{ Kolmogorov-Smirnov $^{\mathrm{a}}$} & \\
& Estadístico & $\mathrm{gl}$ & Sig. \\
\hline Habilidades lingüísticas & 0,158 & 148 & 0,000 \\
Comprensión de lectura & 0,160 & 148 & 0,000 \\
\hline
\end{tabular}

Fuente: Prueba de Kolmogorov Smirnov. 
Jesús Wiliam Huanca-Arohuanca; Meredith Lency Asqui Manzano; Dometila Mamani Jilaja;

Humberto Mamani-Coaquira; Pedro Carlos Huayanca Medina, y Francisco Charaja Cutipa

\section{Decisión estadística}

La significancia de la Prueba de Kolmogorov Smirnov resulta Sig. $=0,000$ lo cual es menor a 0,01 ; razón para aceptar la hipótesis alterna, de tal manera que los datos no siguen la distribución normal; siendo esta prueba altamente significativa.

Por consiguiente, no es viable el análisis de correlación paramétrica y se optó transformar los datos en una escala de valoración cualitativa de carácter ordinal con la siguiente escala de valoración: Pésimo $=\mathbf{1}$, deficiente $=2$, regular $=3$, bueno $=4 \mathrm{y}$ excelente $=5$, para lo cual se aplicó la Prueba de Spearman, adecuada para las características de este tipo de datos.

Prueba de Hipótesis de correlación de Spearman:

a) Hipótesis que se planteó:

Ho: $\mathbf{S x y}=0$ No existe un determinado grado de correlación

Ha: $\mathbf{S x y} \neq 0 \quad$ Existe un determinado grado de correlación

b) Nivel de significancia de probabilidad es: 0,01 .

c) Margen de error considerado: $\alpha=0.05$

d) La prueba de correlación de Spearman

Tabla 12. Correlación entre las Habilidades Lingüísticas y la Comprensión de Lectura.

\begin{tabular}{lllll}
\hline & & \multicolumn{1}{c}{ CORRELACIONES } & \\
& & & $\begin{array}{l}\text { Habilida-des } \\
\text { Lingüísti-cas }\end{array}$ & $\begin{array}{l}\text { Compren-sión } \\
\text { de Lectura }\end{array}$ \\
\hline $\begin{array}{l}\text { Rho de } \\
\text { Spearman }\end{array}$ & $\begin{array}{l}\text { Habilidades } \\
\text { Lingüísticas }\end{array}$ & Coeficiente de correlación & 1,000 & $0,616^{* *}$ \\
& Sig. (bilateral) & $\cdot$ & 0,000 \\
& N & 148 & 148 \\
& $\begin{array}{l}\text { Comprensión } \\
\text { de Lectura }\end{array}$ & Coeficiente de correlación & $0,616^{* *}$ & 1,000 \\
& Sig. (bilateral) & 0,000 & $\cdot$ \\
& $\mathrm{N}$ & 148 & 148 \\
\hline
\end{tabular}

Fuente: Coeficiente de Correlación de Spearman.

\section{Decisión estadística}

El coeficiente de correlación es Rho= 0.616*; ese resultado según la escala de valores de correlación de Spearman, indica que la correlación entre las variables de investigación es positiva considerable, aceptando la hipótesis alterna; donde la probabilidad de la significancia es 0.01 , el cual se considera "altamente significativo". Este resultado significa que el nivel de desarrollo de las habilidades lingüísticas (saber hablar, saber escribir, saber leer y saber escuchar) de los estudiantes investigados está relacionados o asociados en un $61.6 \%$ con los niveles de comprensión de lectura, quiere decir que, cuanto mejor es el desarrollo de las 
Jesús Wiliam Huanca-Arohuanca; Meredith Lency Asqui Manzano; Dometila Mamani Jilaja;

Humberto Mamani-Coaquira; Pedro Carlos Huayanca Medina, y Francisco Charaja Cutipa

habilidades lingüísticas de los estudiantes, mejores resultados se lograrán en la comprensión de textos. Puesto que, las variables actúan conjuntamente en los estudiantes de la IES. "Politécnico Huáscar" de Puno.

En términos más finitos, existe un alto grado de correlación entre el desarrollo de las habilidades lingüísticas y la comprensión de lectura en los estudiantes de la IES "Politécnico Huáscar" de Puno, en el año escolar 2018. Sabiendo que, la tabla de coeficiente de correlación de Spearman tiene un valor de Rho $=0.616^{*}$, lo que expresa que las variables de estudio presentan una relación positiva considerable. De tal manera que, mientras las habilidades lingüísticas se desarrollen de manera adecuada, también se evidenciará un nivel positivo en la comprensión de lectura.

Según los datos obtenidos mediante la ficha de observación, en los colegios de Puno evidencian el desarrollo de sus habilidades lingüísticas en el nivel bueno respectivamente, dado que lo resultados hablan por sí mismos: hablar (57.4\%), escribir (60.1\%), leer (41.2\%) y escuchar (67.6\%), lo que indica que existe un desarrollo adecuado de las habilidades lingüísticas.

De los resultados de la prueba de comprensión de textos, los estudiantes investigados demuestran tener una buena escala de comprensión de lectura, en los siguientes niveles: literal (60.1\%), inferencial (51.4\%) y crítico (67.6\%). Lo que señala que el grado de comprensión de lectura es adecuada en los estudiantes altiplánicos.

\section{Discusión}

Cabe destacar que, los resultados guardan relación con lo que sostienen Mata y otros (2007), quienes afirman en su investigación que el nivel de desarrollo de la habilidades lingüísticas y el nivel de comprensión de lectura en los estudiantes presentan una relación significativa en los estudiantes investigados, lo cual es acorde con lo que esta investigación concluye. De tal manera que, los estudiantes que logran desarrollar adecuadamente sus habilidades lingüísticas (saber hablar, saber escribir, saber leer y saber escuchar) podrán lograr un alto nivel de comprensión de lectura.

Así mismo, Saenz y Gonzales (1998) llegaron a las conclusiones de que existe una estrecha relación entre la lectura comprensiva y las habilidades lingüísticas en los alumnos de la preparatoria No. 3 (Nocturna para Trabajadores) según los parámetros de influencia determinante gracias a una gran gama de factores entre los que se encuentran el nivel cultural, la educación y el nivel socioeconómico.

Por otro lado, esos resultados se asemejan al estudio realizado por Guarneros y Vega (2014) donde sostienen que se pudieron describir las relaciones existentes entre el lenguaje oral y lenguaje escrito (habilidades lingüísticas) con la comprensión de lectura en niños de $1^{\circ}$ básico con un promedio de edad de 6 años 8 meses, donde 51 escolares poseían Trastorno Específico del Lenguaje y el otro grupo estaba constituido por 53 niños con desarrollo típico de lenguaje. Llegando a la conclusión de que un adecuado desarrollo del lenguaje oral por medio del aprendizaje de la lectura y la escritura desde edades tempranas, y a su vez, adquirir la lectura y la escritura son favorables de manera notable en los estudiantes de dicha investigación. Es menester mencionar además que esos resultados se asemejan al estudio realizado por Coloma y otros (2015), cuando en su 
Jesús Wiliam Huanca-Arohuanca; Meredith Lency Asqui Manzano; Dometila Mamani Jilaja;

Humberto Mamani-Coaquira; Pedro Carlos Huayanca Medina, y Francisco Charaja Cutipa

estudio demostraron que existe relación entre la comprensión lectora y habilidades lingüísticas (léxico, conciencia fonológica y discurso narrativo) y su decodificación en escolares con Trastorno Específico del Lenguaje (TEL). Ya que los resultados confirman que la decodificación es una habilidad central para la comprensión lectora. Además, el nivel léxico aparece como la única lingüística que se asocia a la lectura comprensiva en el aprendizaje lector inicial de los niños con Trastorno Específico del Lenguaje.

Ciertamente, los resultados del presente estudio se relacionan a los hallazgos de la investigación realizada por Alanya (2012), cuando concluye que existe una correlación moderada entre la actitud emprendedora y las habilidades sociales en los estudiantes del Quinto de Secundaria de una Institución Educativa del distrito del Callao; así mismo, en sus dimensiones: capacidad de realización, capacidad de planificación con las habilidades sociales existe una correlación alta y en la capacidad de relacionarse socialmente con las habilidades sociales existe una correlación moderada. Además, Padilla y otros (2008) señalan que la competencia o habilidad comunicativa se define como la capacidad que el discente adquiere de saber usar con propiedad una lengua llevándole a distinguir las diversas situaciones sociales posibles. En consecuencia, las habilidades lingüísticas son las competencias, destrezas o capacidades para usar el lenguaje apropiadamente en las diversas situaciones sociales, así como también, en el acto de la comprensión de textos y la lectura, la cual debería ser fuente de placer y nunca una actividad obligatoria y enfrentada como una imposición; la lectura debe comenzar a ser sugerida lo más pronto posible en el proceso de formación de los estudiantes.

De la prueba de comprensión de textos se sostiene que la mayoría de los estudiantes investigados logran recuperar información del texto sin mucha dificultad; sin embargo, en las preguntas donde desarrollan el nivel inferencial y crítico se comprueba cierta dificultad. Si los resultados de la investigación en la Institución Politécnica de Puno son positivos, entonces surge la interrogante esencial y general en todas las esferas educacionales dPerú y la región en mención: ¿por qué el $53 \%$ de los estudiantes a los 10 años no puede leer ni entender un texto simple, a pesar de que en su gran mayoría tienen acceso a la educación? Seguramente la pobreza de aprendizajes mencionado por Jaime Saavedra (2020) podría ser una de las tantas respuestas sobre la problemática educacional altiplánica o es que los ECE no son realmente objetivos a la hora de ser aplicados (Huanca-Arohuanca, 2020b). Por ahora, solo queda instaurar a todos los niveles las habilidades lingüísticas en aras de mejorar la comprensión lectora de los estudiantes puneños.

\section{CONCLUSIONES}

Todo parece indicar que la problemática expuesta en los acápites precedentes, pone a flote el desarrollo de las cuatro habilidades lingüísticas como; saber hablar, saber leer, saber escribir y saber escuchar. Todos ellos, de manera directa o indirecta contribuyen a lograr una comprensión más adecuada de la lectura en los estudiantes del altiplano peruano. Así mismo, favorecen para el uso de un lenguaje apropiado en diversas situaciones sociales como; destrezas de reflexión, la toma de conciencia de las propias capacidades y 
recursos, la memorización, la argumentación y la síntesis de las ideas propias y ajenas que favorecen el aprendizaje de los estudiantes materia de análisis.

En definitiva, el desarrollo adecuado de las habilidades lingüísticas contribuye decisivamente al desarrollo competente de la comprensión lectora de los estudiantes, porque como se ha observado, los escolares presentan una serie de percances gnoseológicos; entendida como conjunto de destrezas para las relaciones societales, la convivencia, formación integral, el aprendizaje significativo, el pensamiento crítico y el entendimiento entre una determinada comunidad educativa y la civilización holística.

\section{REFERENCIAS}

Alanya, S. B. (2012). Habilidades sociales y actitud emprendedora en estudiantes del quinto de secundaria de una Institución Educativa del distrito del Callao [Universidad San Ignacio de Loyola]. http://repositorio.usil.edu.pe/bitstream/1 23456789/1080/1/2012_Alanya_Habilida des-sociales-y-actitud-emprendedora-enestudiantes-del-quinto-de-secundaria-deuna-institucion-educativa-del-distrito-delCallao.pdf

Aliaga, Y. (2012). Comprensión lectora y rendimiento académico en comunicación de los alumnos del Segundo Grado de una Institución Educativa de Ventanilla. Universidad San Ignacio de Loyola.

Andina. (2020, June 3). Las tres regiones del sur peruano obtuvieron los mejores resultados en la evaluación del Minedu en 2019. Andina. https://andina.pe/agencia/noticia-tacnamoquegua-y-arequipa-lideran-mejoraaprendizajes-a-escala-nacional800056.aspx\#: :text="Tacna\%2C

Moquegua y Arequipa son,en estos resultados como Huánuco.

Barón, L., y Müller, O. (2014). La Teoría
Lingüística de Noam Chomsky: del Inicio a la Actualidad. Lenguaje, 42(2), 417-442. http://www.scielo.org.co/pdf/leng/v42n2 /v42n2a08.pdf

Bustamante, G. (2010). Competencia Lingüística y Educación. Folios, 31, 81-90. http://www.scielo.org.co/pdf/folios/n31/ n31a06.pdf

Canaza-Choque, F. A., y Huanca-Arohuanca, J. W. (2019). Disputas por el oro azul: gobernanza hídrica y salud pública. Rev. Salud Pública, 21(5), 1-7. https://doi.org/10.15446/rsap.V21n5.796 46

Cárdenas-Espinoza, K., y Guevara-Benítez, Y. (2013). Comprensión lectora en alumnos de secundaria. intervención por niveles funcionales. Journal of Behavior, Health $y$ Social Issues, 5(1), 67-83. https://doi.org/10.5460/jbhsi.v5.1.38727

Coloma, C. J., Sotomayor, C., De Barbieri, Z., y Silva, M. (2015). Comprensión lectora, habilidades lingüísticas y decodificación en escolares con TEL. Revista de Investigación En Logopedia, 1, 1-17. https://www.redalyc.org/pdf/3508/3508 41434001.pdf

Economía y Negocio. (2019, May 9). La realidad de la comprensión lectora en nuestro país. Economía y Negocio. http://economiaynegocio.com/2019/05/0 9/la-realidad-de-la-comprension-lectoraen-nuestro-pais/

Escurra, L. M. (1998). Siegel, S. y Castellan, N. J. (1995). Estadística no paramétrica, aplicada a las ciencias de la conducta. 4a. edición. México: Editorial Trillas. Persona, 001, 195-196. https://doi.org/10.26439/persona1998.n0 01.1715

García Bellido, R., González, J., y Jornet, J. M. (2010). SPSS: pruebas no paramétricas Kolmogorov Smirnov. Grupo de Innovacion Educativa. Universidad de Valencia, 1-5. https://www.uv.es/innomide/spss/SPSS/ SPSS_0802A.pdf

Guarneros, E., y Vega, L. (2014). Habilidades lingüísticas orales y escritas para la lectura 
y escritura en niños preescolares. Avances En Psicología Latinoamericana, 32(1), 2135.

https://doi.org/10.12804/apl32.1.2014.02

Gutierrez, L., y Sanz, J. (2018). Talleres con niñas y niños en la formación inicial del profesorado: Superación del modelo transmisivo a partir de una experiencia Universidad-Escuela. Profesorado. Revista de Currículum y Formación Del Profesorado, 22(1), 301-324.

Gutiérrez, R. (2018). Efectos de la lectura compartida y la conciencia fonológica para una mejora en el aprendizaje lector. Revista Complutense de Educación, 29(2), 441-454. https://doi.org/10.5209/RCED.52790

Hernández, R., Fernández, C., y Baptista, P. (1991). Metodología de la investigación. McGraw-Hill.

Hernández, R., Fernández, C., y Baptista, P. (2014). Metodología de la investigación (6a ed.). McGRAW-HILL.

Huanca-Arohuanca, J. W. (2019). El despertar de una nueva era: colonización y camino hacia la revolución. Revista Revoluciones, 1(1), 1-3. http://revistarevoluciones.com/index.php /rr/article/view/1/2

Huanca-Arohuanca, J. W. (2020a). Caleidoscopio social al Covid-19: pánico y desesperación en tiempos de aislamiento. Revista Universidad y Sociedad, 12(6), 226231.

https://rus.ucf.edu.cu/index.php/rus/artic le/view/1836

Huanca-Arohuanca, J. W. (2020b). Contrahegemonía y la lucha por la educación en el sur dPerú. Editorial Académica Española.

Huanca-Arohuanca, J. W. (2020c). Cuando la muerte nos mira con deseo de llevarnos al tártaro. Revista Revoluciones, 2(2), 1-5. https://doi.org/10.35622/j.rr.2020.02.001

Huanca-Arohuanca, J. W. (2020d). Retropías y distopías de la educación en Puno. Revista Helios, 4(1), 270-271. https://doi.org/10.22497/Helios.41.4116
Huanca-Arohuanca, J. W. (2021). Narrativas de guerra y resistencia: participación de la mujer austral dPerú en la Guerra del Pacífico. Encuentros. Revista de Ciencias Humanas, Teoría Social y Pensamiento Crítico, 13, 53-62.

Huanca-Arohuanca, J. W., Butrón, S. B., Supo, L. A., y Supo, F. (2020). Evaluación y monitoreo de la calidad ambiental del agua en el proyecto sistema de riego Canal N, provincia de Melgar - Puno, Perú. Ciencia y Desarrollo, 19(1), 88-96. http://revistas.unjbg.edu.pe/index.php/cy d/article/view/936/1062

Huanca-Arohuanca, J. W., y Canaza-Choque, F. A. (2019). Puno: Educación rural y pensamiento crítico. Hacia una educación inclusiva. Revista Helios, 3(1), 97-108. https://doi.org/10.22497/Helios.31.3106

Huanca-Arohuanca, J. W., Canaza-Choque, F. A., Escobar-Mamani, F., y Ruelas, D. (2020). En defensa del pluralismo latinoamericano: las esferas de la justicia y la igualdad compleja en Michael Walzer. Un dilema pendiente por atender. Chakiñan. Revista de Ciencias Sociales y Humanidades, 11, 92103. https://doi.org/10.37135/chk.002.11.07

Huanca-Arohuanca, J. W., Escobar-Mamani, F., y Supo-Condori, F. (2020). Democratización y acceso a la información de la sociedad civil: un análisis diacrónico a la gestión 2015 - 2018 del Gobierno Municipal Puno - Perú. Sciéndo, 23(2), 93103.

https://doi.org/10.17268/sciendo.2020.0 14

Huanca-Arohuanca, J. W., y Geldrech, P. (2020). Planificación educativa y gestión pedagógica-estratégica-operacional en las instituciones del nivel inicial en el sur dPerú. Revista Conrado, 16(76), 369-376. https://conrado.ucf.edu.cu/index.php/con rado/article/view/1497

Huanca-Arohuanca, J. W., y Pilco, N. (2021). Acciones revolucionarias en Ámérica Latina: Puno y el Alto Perú durante el 
proceso de independencia (1809-1825). Chakiñan. Revista de Ciencias Sociales y Humanidades,

14. https://doi.org/10.1590/SciELOPreprints. 1364

Madero, I. P., y Gómez, L. F. (2013). El proceso de comprensión lectora en alumnos de tercero de secundaria. Revista Mexicana de Investigacion Educativa, 18(56), 113-139. http://www.scielo.org.mx/pdf/rmie/v18n 56/v18n56a6.pdf

Martínez, R. M., Tuya, L. C., Martínez, M., Pérez, A., y Cánovas, A. M. (2009). El coeficiente de correlación de los rangos de Spearman caracterización. Revista Habanera de Ciencias Médicas., 8(2). https://www.redalyc.org/articulo.oa?id=1 80414044017

Mata, S., Gallego, J., y Mieres, C. (2007). Habilidades lingüísticas y comprensión lectora. Una investigación empírica. Bordón. Revista de Pedagogía, 59(1), 153166.

https://recyt.fecyt.es/index.php/BORDON /article/view/36841

Padilla, D., Martínez, M. del C., Pérez, M. T., Rodríguez, C., y Miras, F. (2008). La competencia lingüística como base del aprendizaje. International Journal of Developmental and Educational Psychology, 1 , 177-184. https://www.redalyc.org/pdf/3498/3498 32317019.pdf

Pérez, J. (2005). Evaluación de la comprensión lectora: dificultades y limitaciones. Revista de Educación, 121-138. http://www.ince.mec.es/revistaeducacion /re2005/re2005_10.pdf

Prieto, L. Á. (2012). Evaluación de la
Competencia lingüística en alumnos de $4^{\circ}$ de Primaria [Universidad de León]. https://buleria.unileon.es/bitstream/hand le/10612/2157/tesis_541687.pdf?sequenc $\mathrm{e}=1$

Ramos, C. (2006). Elaboración de un instrumento para medir comprensión lectora en niños de octavo año básico. Onomázein, 14, 197-210. http://onomazein.letras.uc.cl/Articulos/14 /7_Ramos.pdf

Reyzábal, V. (2012). Las competencias comunicativas y linguísticas, clave para la calidad educativa. Revista Iberoamericana Sobre Calidad, Eficacia y Cambio En Educación, 10(4), 63-77. https://revistas.uam.es/reice/article/view /2988/3208

Saavedra, J. (2020, January 6). Aprendizajes: podemos estar mejor. Noticias Gestión Perú, 1-9. https://gestion.pe/blog/politicas-publicaspara-el-desarrollo/2020/01/aprendizajespodemos-estar-mejor.html/?ref=gesr

Saenz, G. A., y Gonzales, M. del R. (1998). La lectura comprensiva y las habilidades linguisticas en el estudiante de preperatoria nocturna [Universidad Autónoma de Nuevo León].

http://eprints.uanl.mx/642/1/102012365 5.PDF

Velázquez, T., Rivera-Holguín, M., y Custodio, E. (2017). Aportes y reflexiones de la formación en psicología comunitaria PUCP en tres regiones de Perú. Revista de Psicología, 35(1). https://doi.org/10.18800/psico.201701.0 07 